\title{
Italique
}

XX $\mid 2017$

Varia

\section{L'amour créateur}

\section{Étienne Barilier}

\section{(2) OpenEdition}

Journals

Édition électronique

URL : http://journals.openedition.org/italique/469

DOI : $10.4000 /$ italique.469

ISSN : 1663-4438

\section{Éditeur}

Librairie Droz

\section{Édition imprimée}

Date de publication : 1 octobre 2017

Pagination : 293-308

ISBN : 978-2-600-05818-6

ISSN : 1423-3983

\section{Référence électronique}

Étienne Barilier, «L'amour créateur », Italique [En ligne], XX | 2017, mis en ligne le 01 octobre 2019, consulté le 23 janvier 2020. URL : http://journals.openedition.org/italique/469 ; DOI : 10.4000/italique. 469

(c) Tous droits réservés 
Ét TENNE B A R I LIER

L'A M O U R C É A T E U R 



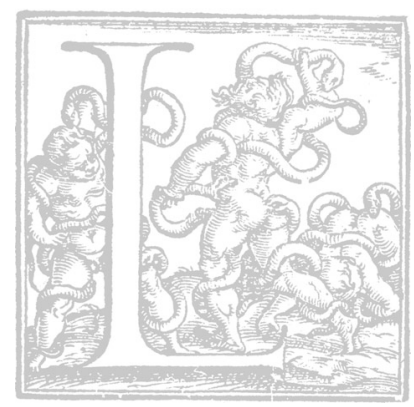

e I8 avril I485, à Rome, sur la via Appia, près du monastère de Santa Maria Nuova, des maçons lombards qui travaillaient, ô blasphème, à démolir un tombeau antique pour en réemployer le marbre, découvrirent un sarcophage qui, ouvert, leur révéla une manière de miracle: le corps intact d'une jeune fille. 'L'bumaniste florentin Bartolomeo della Fonte, ${ }^{2}$ dans une lettre à un ami, la décrit en ces termes: "Son visage était lumineux comme si elle avait été ensevelie le jour même». ${ }^{3}$ Un autre témoin de cette apparition écrit qu'elle était «si belle et parfaite que l'on ne peut guère l'écrire on le dire, et que si on le disait et l'écrivait, ceux qui ne l'ont pas vue ne le croiraient pas». ${ }^{4}$

Celle qu'on appela aussitôt la «puella Romae» (la jeune fille de Rome; entendez: de la Rome antique), fut transportée au Capitole, pour être exposée aux yeux de la foule extasiée. Mais après un ou deux jours, sur l'ordre du pape Innocent VIII, la jeune morte, dont certains juraient qu'il s'agissait de Tullia, la fille même de Cicéron, fut enlevée à la vénération publique et réenterrée près de la Porta Pinciana. Si son corps miraculeusement préservé fut ainsi soustrait aux regards, c'était peutêtre parce que les autorités ecclésiastiques pouvaient craindre que ce miracle ne suscitât une adoration par trop païenne. Mais c'était peutêtre pour la raison contraire, si je puis dire: afin d'éviter que le miracle ne se fane. Car au contact de l'air, les chairs commençaient de s'altérer, les lignes du visage de se brouiller.' Malgré de nombreuses recherches, qui se sont poursuivies jusqu'à nos jours, on n'a d'ailleurs jamais su qui était cette jeune fille et pourquoi elle avait été embaumée selon des techniques proches de celles des anciens Égyptiens. ${ }^{6}$

Mais peu importe. Jakob Burckhardt, qui dans son fameux ouvrage sur la Civilisation de la Renaissance en Italie, évoque ce saisissant épisode, insiste sur un point - sur le point capital: aux yeux des spectateurs du $X V^{\star}$ siècle, le corps et le visage de cette jeune morte ne pouvaient qu'être beaux, et surtout plus beaux que tout visage et tout corps vivant en I485, tant on était persuadé que l'Antiquité, dans ses cuvres et jusque dans ses exemplaires humains, si j'ose ainsi m'exprimer, était supérieure aux temps présents. ${ }^{7}$ Supérieure moralement et physiquement. D'ailleurs une épigramme latine, composée peu après la 
découverte, et mise dans la bouche de la morte anonyme, dit en substance: sortie de ma tombe, je ne vois plus rien, dans la Rome actuelle, qui égale, en justice et en piété, la Rome que j'ai connue. ${ }^{8}$

La Renaissance italienne, ce grand élan de passion pour l'bumain, n'est évidemment pas datée, chronologiquement, du I8 avril I485. Mais à ce jour, avec cette exhumation de la jeune Romaine, elle a commencé symboliquement, ou pour mieux dire, elle s'est incarnée. Car la Renaissance italienne commence bien par là: par un amour éperdu, absolu, du monde antique, par la certitude que le passé grec et romain est ce qu'il $y$ a de plus vivant au monde; qu'il porte la lumière physique et morale sans laquelle nous sommes des avengles. La découverte de la «puella Romae» fut un événement bien réel, historiquement attesté. Mais si ce fut un événement, c'est parce que la présence vivante, et stupéfiante de vie, d'un corps antique et d'une beauté d'autrefois répondait à un désir profond de toucher la chair même de l'Antiquité, de suivre les courbes de son visage, d'habiter son temps; ce désir, la découverte du I 8 avril I485 le comblait de façon merveilleusement littérale. Il ne nous étonnera pas d'apprendre que parmi la foule des adorateurs de la jeune Romaine se trouvaient des peintres. D'ailleurs, nous gardons un dessin de son corps et surtout de son visage, dessin qui était joint à la lettre de Bartolomeo della Fonte, et sur l'aspect duquel je reviendrai. ${ }^{\text {? }}$

Si cet amour absolu du passé antique trouve ici son expression presque effrayante à force d'être immédiate, le $X V^{\mathcal{P}}$ siècle italien nous en donne bien d'autres preuves, non moins éloquentes. Dans sa biographie de Laurent de Médicis, écrite au début du XVT siècle, l'bistorien Niccolò Valori raconte que le maître de la République de Florence avait toujours soubaité posséder un buste de Platon. Entendons: un vrai buste du vrai Platon, sculpté quand le modèle était vivant. Or un beau jour, nous assure le chroniqueur, ce rêve est devenu réalité: on fit cadeau à Laurent du buste tant cherché. On l'avait découvert à Athènes, et dans les ruines mêmes de l'Académie. ${ }^{10}$ Certes, ce n'était pas tout à fait le cadavre embaumé de ce philosophe illustre entre tous; mais à défaut, son véritable portrait, découvert sur le site sacré où l'auteur du Banquet et $d u$ Phèdre méditait en levant les yeux vers le ciel des Idées éternelles. Il va sans dire, bélas, que cette prétendue authenticité n'était qu'un mythe. Mais qu'on ait fabrique ce mythe avec ferveur exprime assez. à quel point fut vive, dans la Florence de Laurent le Magnifique, la 
passion de l'Antiquité grecque, et le désir religieux de sa présence réelle. Il s'agissait bien d'un culte, en effet. Après le règne de Laurent, les disciples de l'intransigeant Savonarole, dénonciateurs du paganisme ambiant, assurèrent que le philosophe platonolâtre Marsile Ficin avait allumé, devant ce portrait de Platon, une lampe votive, comme on le fait sur un autel. La renaissance platonicienne n'est pas simplement un retour d'intérêt, si passionné soit-il, pour un Platon mort, c'est la volonté farouche que Platon soit vivant, puisqu'il est éternel.

Ce n'est d'ailleurs pas un hasard si le philosophe byzantin Gémiste Pléthon qui, venu de Constantinople, forma les humanistes florentins, à commencer par Marsile Ficin, à la langue grecque et à la pensée de Platon, était partisan d'instaurer dans ce qu'il appelait encore l'Empire romain un platonisme philosophique et politique, et de rejeter purement et simplement le christianisme. Platon, tel quel, n'appartenait pas au passé mais bien au présent, et au futur.

Pour donner une dernière illustration de cet amour absolu des Anciens, et de ses effets sur la création renaissante, j'évoquerai une deuxième jeune fille, non pas une revenante de la Rome antique, mais une baute figure de la Florence médicéenne: la très remarquable Alessandra Scala (I475-I506), qui se rendit célèbre pour avoir tenu, d'une manière incomparable, le rôle d'Électre dans la pièce éponyme de Sophocle, jouée en grec ancien bien entendu. Bref, Alessandra Scala rendait Électre présente, rendait Électre à sa présence. Et non contente d'être actrice, elle fut poétesse, composant des vers en grec ancien, notamment pour répondre aux éloges que lui adressait, dans la même langue, son compatriote et confrère amoureux, Angelo Poliziano. ${ }^{\text {I }}$

Telle fut la passion de la Renaissance pour la langue et la culture de Sophocle et d'Homère. Au travers de ces trois exemples: le corps intact de la jeune Romaine, le buste du vrai Platon objet de culte, et la poétesse et actrice Alessandra Scala récitant et écrivant des vers grecs, j'ai voulu souligner que la Renaissance italienne a eu pour condition - insuffisante mais nécessaire - un amour, un désir éperdus de retrouver non pas le passé mort, mais le passé vivant, de se couler en lui, de le couler en soidonc, peu ou prou, de l'imiter.

Mais qu'en est-il à cet égard des artsplastiques? Eh bien, il n'estpas douteux que la théorie de la peinture comme mimésis, c'est-à-dire imitation, théorie que l'on trouve aussi bien chez. Leon Battista Alberti que chez. Lorenzo Ghiberti ${ }^{2}$ ou Léonard de Vinci, a des origines platoniciennes et 
néoplatoniciennes, et s'est vue stimulée, pour ne pas dire éveillée, par la redécouverte, dans leur langue originale, des cuvres grecques.

Bien sûr, il ne s'agit pas alors d'imiter la peinture grecque, que d'ailleurs on ne connaît que sur la foi de ses témoins antiques, mais d'imiter la leçon des Anciens. Quelles que soient les infinies complexités de la notion d'«imitation», il reste, aux origines de la Renaissance picturale, cette idée que ce qu'il y a de plus réel, ce qu'il faut atteindre par l'art, que ce soit la «nature» ou l'«idée», est une réalité préalable, antérieure, parfaite sans nous, vivante hors de nous, et qu'il faut l'atteindre à force de ferveur, de labeur et d'amour. Lorsque Léonard écrit, dans son Trattato della pittura, que "l'art de la peinture a un caractère divin, en ce que l'esprit du peintre se transmue à la ressemblance de l'Esprit de Dieus, ${ }^{3}$ il entend bien que le modèle de la peinture est une essence éternelle. Et de cette essence éternelle, on a vu que le passé antique est la plus vivante image, même si de vains esprits le croient mort et enterré.

L'élan éperdu vers un passé qu'on éprouveplus vivant que soi-même et que son éphémère présent, telle est la condition nécessaire de la Renaissance. Nécessaire mais insuffisante, bien sûr. Car enfin, si vraiment les hommes de cette époque, à force de scrupuleux amour, n'avaient fait que recopier et répéter le passé antique, si beau et si riche puisse-t-il être, ils n'auraient exactement rien fait. Leurs cuvres n'auraient été que de vains pastichesce qu'à vrai dire elles sontparfois, chezles auteurs mineurs. Leur beauté de conserve se seraitfanée àpeine surgie au grand air de la vie, comme devait se faner la beauté de la «puella Romae». Non, bien sûr, les hommes de la Renaissance ont manifesté un pouvoir créateur et novateur à la mesure de leur passion pour l'antique; ils sont allés, si je puis paraphraser Baudelaire, au fond du très ancien pour trouver du nouveau. Fût-ce sans le vouloir. Carne l'oublions pas, même si l'on trouve le mot de rinascita sous la plume de Pétrarque, la Renaissance, de manière générale, ne savait pas qu'elle était la Renaissance.

Ce qu'il faut avoir présent à l'esprit, c'est que son amour de l'Antiquité fut d'abord amour de la vie, d'une vie fraîche et neuve, d'une Vita nova pour parler comme Dante, et que, comme tout amour, ce sentiment a façonné peu ou prou son objet. Et dès lors, le Quattrocento italien a donné, à sa vision de l'bomme et du monde, une inflexion nouvelle; une inflexion seulement, mais décisive, et dont nous vivons encore aujourd'bui les effets, dont nous cueillons encore aujourd'bui les fruits. 
Chez les peintres, quelque chose, dans le visage et le corps de l'homme et de la femme, s'anime, une palpitation surgit en eux, une respiration les soulève, qui les fait plus mortels et plus vivants, de même que la peinture des paysages, moins hiératique, moins impassible qu'elle n'avait été jusqu'alors, devient la représentation d'une nature animée, traversée de lumières passagères et d'oiseaux mortels, obéissant au rythme des saisons, et les courbes des collines toscanes répondent aux courbes des visages des Vierges à l'Enfant. Car l'injonction d'«imiter la nature», qui fut celle de la peinture renaissante, conduisit à ne plus faire de cette nature le seul cadre symbolique ou stylisé d'une représentation sacrée, comme dans la peinture du Moyen-Âge, mais à la restituer telle que les hommes la voient et la vivent. D'où l'invention et l'introduction de la perspective. Le mot est bien choisi: la Renaissance voit désormais toute chose dans la «perspective» de l'homme.

La transformation que cela fait naître peut paraître modeste, et la transition, de Duccio à Giotto et de Giotto à Filippo Lippi puis à Botticelli, est subtile et sans heurt. Mais à son terme, c'est l'éclatement $d u$ Printemps botticellien, que Duccio n'aurait jamais pu concevoir. Qu'est-ce qui a changé? Quand cela a-t-il changé? Comment? Comment le corps spiritualisé, majestueux, sacralisé, vêtu d'éternité, comme empesé par l'autre monde, est-il devenu le corps palpitant, charnel, habité par le souffle et le sourire de la vie simplement humaine, dans une nature apprivoisée, arpentée, habitable? Cette métamorphose dont on ne pourra bien sûr jamais saisir l'instant décisif, est pourtant irrécusable, et continue aujourd'bui de nous enchanter, au sens le plus fort de ce mot. Oui, vraiment, la passion absolue de la Renaissance italienne pour l'Antiquité, ce fut d'abord une passion neuve de la vie dont cette Antiquité témoignait; une passion non d'exprimer l'éternité dans le temps comme avait fait le Moyen-Âge, mais de rendre vie ou de donner vie à ce qui est mortel, en acceptant le temps humain. $\dot{A}$ vrai dire, on ne veut ni ne peut ressusciter le passé; mais on veut et on peut le recréer; trouver, dans l'homme du passé, l'homme présent et futur. C'est cette nouveauté insaisissable et radicale que Jakob Burckbardt a résumée lorsqu'il a parlé de la Renaissance italienne comme d'une époque de la "découverte de l'bomme».

Il est d'ailleurs temps que je le note: le dessin qui nous est parvenu du visage de la «puella Romae», la jeune morte de 1485 , ce dessin ressemble étrangement à celui du visage d'une Vierge du Quattrocento... Et, vous 
l'aurez, compris, la découverte de la «puella Romae», ce fut d'abord et surtout une invention de la «puella Romae», au double sens du mot: au sens de l'histoire sacrée et de l'bistoire de l'art, qui parle d'«invention de la croix», mais aussi au sens commun du mot. Ce qu'on découvre, on l'«invente», c'est-à-dire qu'on le crée.

Coïncidence éclairante, lorsque Boccace évoque la figure de Giotto, il le crédite d'avoir «ramené à la lumière» un art «enseveli». Oui, l'art nouveau est une sortie du tombeau, mais à l'image de la puella Romae, il ressuscite non tel qu'il fut, mais tel qu'on veut qu'il soit: tel qu'en lui-même enfin notre passion le change.

Pour montrer sur l'exemple d'un texte, et d'un texte fameux, ce phénomène de création au ccur même de l'imitation, de novation à la faveur même de la répétition, je voudrais me pencher un instant sur le fameux discours intitulé De la dignité de l'homme, qui devait être prononcé à Rome, sur le lieu même de la déconverte de la «puella Romae», et prononcé en I486, donc tout juste une année après cette découverte. Ce texte, Jakob Burckhardt le qualifie d'un des plus beaux héritages que nous ait laissés la Renaissance. ${ }^{\mathrm{I}}$ Mais toute personne qui se penche sur cette époque de l'Europe ne peut que tomber en admiration devant lui. Quant à moi, il m'avait tant frappé, il y a de cela plus de trente ans, que j'ai consacré tout un roman, Le Dixième ciel, à son auteur, Giovanni Pico della Mirandola. Si j'en reparle aujourd'bui, ce n'est pas seulement pour le bonheur égoïste de retrouver le personnage et l'cuvre qui m'ont habité voilà trois décennies, et qui ne cessent guère de m'accompagner. C'est aussi parce qu'il va nous permettre de saisir sur le vif cette inflexion à la fois légère et décisive qui a fini, de proche en proche, par modifier le visage de l'Europe tout entière, sinon davantage. Ce texte est l'aile de papillon dont le battement fait lever à lui seul les grands vents du renouveau de l'bomme et du monde.

Et pourtant, on pourrait croire à première vue qu'il ne fait que copier, répéter et imiter des textes antérieurs, chrétiens ou païens. Pic de la Mirandole, comme ses contemporains, est passionné d'Antiquité. Sa puissance intellectuelle est hors du commun, mais il semble qu'il se contente de rassembler et de résumer dans son texte tout l'béritage philosophique des Anciens. Néanmoins, dans son résumé, nous allons trouver cette fameuse et légère et décisive inflexion, celle même que nous admirons dans le mystérieux assouplissement des formes de la peinture italienne. Je vous lis ici les phrases-clés du discours Sur la dignité de 
l'homme. C'est Dieu qui parle et qui s'adresse à l'homme après avoir crée le monde et les animaux:

O Adam, nous ne t'avons donné ni siège certain, ni visage propre, ni aucun don particulier, afin que tu détiennes et possèdes, selon tes voeux et ta volonté, le siège, le visage et les dons que tu auras souhaités, toi. [...]. Je t'ai placé sous le pouvoir de ta propre volonté, qui déterminera ta nature. Je t’ai placé au milieu du monde afin que tu puisses aisément le dominer du regard. Je ne t’ai fait ni céleste ni terrestre, ni mortel ni immortel, afin que, maitre de toi-même, tu hérites l'honneur et la charge de te modeler toimême, de te composer la forme que tu auras préférée. Tu pourras dégénérer dans l'animalité des formes inférieures; tu pourras, si ton âme le décide, être régénéré dans les formes supérieures, qui sont divines. ${ }^{15}$

Certains commentateurs, comme le cardinal de Lubac, dans l'ouvrage qu'il a consacré à Pic, ont estimé que ce texte était parfaitement orthodoxe et ne bouleversait guère la vision de l'homme tel que l'avait façonnée la tradition chrétienne. Ce savant n'a pas de peine à montrer que l'éloge de la liberté humaine se trouve déjà chez les Pères de l'Église, par exemple chez. Clément d'Alexandrie ou chez. Grégoire de Nysse, qui, plus de mille ans avant Pic, exaltait la «dignité royale» d'une «âme bumaine maîtresse de son propre vouloir»». ${ }^{16}$ Quant à l'idée de l'animalité et de la divinité, donc de l'ange et de la bête, on la trouve aussi, assez abondamment, chez les Pères. On peut d'ailleurs remonter plus haut encore que les Pères de l'Église: jusqu'à Sophocle et son fameux éloge de l'bomme, proféré par le chour, dans Antigone:

Il est bien des merveilles en ce monde, il n'en est pas de plus grande que l'homme. [...] Il peut prendre la route du mal comme du bien. ${ }^{17}$

Autrement dit, Pic aurait brillamment mais fidèlement copié Sophocle, ${ }^{18}$ comme il aurait copié, brillamment mais fidèlement, les Pères de l'Église. Bref, il les aurait tous imités, dans un beau geste d'amour mimétique.

Mais alors, comment se fait-il que ce texte - que son auteur n'a d'ailleurs pas pu lire à Rome en I486, et qui ne fut pas publié de son vivant - ait à ce point marqué les esprits quand il finit par être connu, et surtout les esprits du XX siècle? Du kantien Ernst Cassirer au marxiste Ernst Bloch, on y a vu l'expression par excellence de la liberté 
de l'bomme moderne, dans un monde résolument laï puis athée. C'était sans doute aller trop vite et trop loin, et le cardinal de Lubac était fondé à tempérer de telles ardeurs. Pourtant, si ce discours de Pic de la Mirandole n'est pas un manifeste marxiste ou kantien, quelque chose de subtilement mais radicalement nouveau s'y trouve bel et bien, cette douce et subtile inflexion qui nous ouvre un monde, qui nous dévoile un borizon tout neuf. Où donc?

Eh! bien, si Pic n'est pas le premier à chanter la liberté de l'homme, il la chante d'une manière qui rend cette liberté plus large et plus vertigineuse qu'elle n'avait jamais été. Pour exprimer que l'homme est libre, il ose affirmer que celui-ci, en naissant, n'est littéralement rien de défini, rien de définissable:

Nous ne t’avons donné ni siège certain, ni visage propre, ni aucun don particulier. [...] Je ne t'ai fait ni céleste ni terrestre, ni mortel ni immortel.

Ces mots-là sont réellement inouïs, et leur nouveauté même a pu, dans une mesure certaine, échapper à leur jeune auteur (car Pic était très jeune quand il écrivit ce texte). ${ }^{19}$ Si l'on y réfléchit bien, «ni siège certain, ni visage propre» est d'une inquiétante étrangeté; cela dit beaucoup plus que: «l'homme est libre»; cela nous ouvre un champ beaucoup plus vaste, presque angoissant d'immensité. Quant à «ni céleste ni terrestre, ni mortel ni immortel), c'est encore plus étrange et ne veut apparemment rien dire, car comment serait-il possible de n'être pas l'un ou l'autre, céleste ou terrestre, mortel ou immortel? L'bomme serait-il alors un mélange des deux? Mais cela, à son tour, n'aurait pas de sens: qu'est-ce qu'un mélange de mortalité et d'immortalité? ${ }^{20}$

Pourtant ces mots du discours de Pic, loin de ne vouloir rien dire, disent d'une manière étonnante que l'bomme n'est rien, c'est-à-dire rien d'assignable ni de définissable, un paradoxe vivant; que l'bomme est tout entier dans sa liberté d'être. Sophocle affirmait que l'homme est capable de tout, dans le bien comme dans le mal. Pic nous fait éprouver que l'homme est l'être potentiel par excellence. Les Pères de l'Église, de leur côté, professaient que l'homme est libre. Pic nous fait découvrir que l'homme est celui en qui surgit la liberté, ou mieux encore, qu'il est le surgissement même de cette liberté, aussi entière qu'elle est littéralement impensable («ni mortel ni immortel»).

Dans les deux cas, il ne s'agit, c'est vrai, que d'une inflexion, et tout le reste du texte de Pic Sur la dignité de l'homme retrouve et rejoint 
assez sagement la pensée orthodoxe de son temps. Mais elle est bien là, cette inflexion. Soudain, même si c'est Dieu qui parle, nous sentons que le personnage absolument central, le foyer du texte, c'est l'bomme, d'où rayonne un mystère indicible et précieux. Nous pouvons surprendre et saisir cette inflexion dans les mots comme nous pouvons découvrir, en comparant une Vierge de Duccio à une Vierge de Filippo Lippi, la chair divine devenir chair féminine, la courbe austère devenir courbe sensuelle, l'éternité devenir le temps, la solennité transcendante devenir grâce humaine - devenir, surtout, visage humain. Toute la Renaissance est visage, toute la Renaissance est assomption de l'bumain. La «dignité de l'bomme» n'a jamais été reconnue à ce point; jamais ne lui fut concédé un tel espace de liberté, et s'il a vocation si radicale à se faire lui-même, comment ne l'aurait-il pas à se peindre lui-même, en sa simple et unique, irréductible et glorieuse bumanité?

Or c'est bien ce qu'il a fait dans ses chefs-d'cuvre picturaux. Le plus bel exemple que je connaisse de cette nouvelle incarnation de l'homme dans la peinture italienne de ce temps se trouve peut-être à Spello, en Ombrie, et c'est l'ensemble des peintures que réalisa Bernardino Pinturicchio, exactement à la charnière du $X V^{e}$ et du XVI siècles, pour la chapelle Baglioni de l'église Saint-Marie Majeure. Cette chapelle est souvent surnommée, à combien juste titre, la «capella bella». Ses fresques représentent l'Annonciation, la Nativité et Jésus au milieu des docteurs. Les figures de la Vierge, de l'Enfant et de l'Ange annonciateur, merveilles d'or et de finesse, sans oublier celle de Dieu même, qui ne rechigne pas à revêtir un bienveillant visage, accompagnent harmonieusement celle des bumains dont la rudesse terrienne et la simplicité de paysans noueux sont dignes du Décaméron. Et le tout est surmonté de sibylles hiératiques, pourtant aussi gracieuses que la Vierge! On ne peut imaginer plus de spiritualité libérale, plus de bonbeur à dire cette spiritualité dans la beauté, sans jamais oublier la plus bumble bumanité. On ne peut admirer recueil plus harmonieux du paganisme dans la vision chrétienne. On ne peut concevoir accueil plus généreux à l'esprit comme au corps, et tout cela dans une atmosphère de rêve qui pourtant ne nous fait pas quitter la terre. C'est la pureté de Fra Angelico et la carnalité de Raphaël. La Renaissance italienne, la voilà tout entière.

Mais il faut aussi voir - et c'est de cela que je voudrais encore vous parler un instant avant de conclure - que la médaille a son revers, et la 
liberté sa souffrance. Si la Renaissance est à bien des égards le sacre de l'humain, la promotion de la vie d'ici-bas, l'émancipation du temps, secouant doucement la tutelle de l'éternité comme ses madones secouent au vent leurs cheveux d'or, cela signifie aussi que les humains connâेtront, plus peut-être que leurs prédécesseurs, les angoisses du choix, du vide et de la mort. Les angoisses de l'bumain rien qu'bumain. Bien entendu, je ne veux pas dire que l'bumanité du Moyen-Âge, et du Moyen-Âge finissant particulièrement, n'ait pas connu les affres de la condition bumaine et du trépas! Au contraire, et Johan Huizinga, dans son fameux Automne du Moyen-Âge, souligne même «qu'aucune autre époque à son déclin n'a donné autant d'accent et de pathos à l'idée de la mort». ${ }^{21}$ Mais cette idée était alors liée à un rejet horrifié du monde et de la chair, en même temps qu'à une sorte de fascination pour le macabre. "L'émotion», dit encore Huizinga, «se pétrifiait [alors] dans la représentation réaliste de la mort hideuse et menaçante». Une mort à laquelle le corps ne pouvait échapper, mais à laquelle l'âme échapperait pourvu qu'on la remît à Dieu.

Or l'effroi médiéval de la mort va devenir, à la Renaissance, l'angoisse de la mort, inséparable, oui, de l'angoisse de la liberté. Le corps et l'âme sont désormais embarqués dans les mêmes joies et les mêmes douleurs, ils se sentent également menacés comme ils sont également glorifiés. L'être bumain n'est plus une âme dans un corps, c'est un corps-âme palpitant. La passion sensuelle et l'élan spirituel ont même destin. Ou si l'on préfere, le spirituel même devient passion, et passion douloureuse. C'est Botticelli peignant la Naissance de Vénus tout en illustrant la Divine comédie - car il semble qu'il ait travaillé aux deux cuvres presque conjointement.

Mais l'exemple le plus flagrant et le plus formidable de cette nouvelle conscience, souffrante, du monde et de l'homme, de cette angoisse spirituelle de la mort de l'âme, plus terrible que l'effroi médiéval de la mort du corps, on le trouve chez celui qui vécut assez. longtemps - mais de quelle vie tourmentée - pour embrasser à lui seul une très large partie de la période que nous appelons aujourd'bui Renaissance. J'ai nommé bien sûr Michel-Ange (I475-I564). Nul besoin de longs discours. Si l'on compare sa Pietà de jeunesse, celle qui se trouve à Saint-Pierre de Rome, avec la Pietà Rondanini, au château Sforz̧a de Milan, cuvre inachevée à laquelle il travaillait à la veille de sa mort, on voit, on sent que la première, malgré toute la douleur qu'elle exprime, n'est encore, 
précisément, qu'expression de la douleur. La seconde est la douleur même, et la mort même; elle est silence, absence, détresse, déréliction. La première est la mort de la chair, la seconde est la mort de l'âme.

Cette douleur extrême, cette douleur spirituelle, cette torture d'une âme dans un corps, d'une âme qui se sait menacée dans sa vie propre, se lit aussi dans les dernières cuures, bouleversantes, de Michel-Ange poète. Voilà décidément qu'est né, avec l'homme de la Renaissance, l'homme moderne: l'homme libre, donc angoissé, et pris dans un combat spirituel dont l'issue est au moins incertaine.

Je veux conclure et ne saurais terminer sur une note si sombre. Quand on parle de Renaissance, c'est pour y croire, et croire qu'à tout prendre, l'assomption de l'bumain vaudra toujours mieux que sa sujétion, son écrasement ou son inexistence. Mais au fait, que signifie, aujourd'bui, croire à la Renaissance? Dans le monde tel qu'il va, tel qu'il souffre et s'égare, une nouvelle ère aussi féconde que le Quattrocento italien est-elle encore possible, ou simplement concevable? Voilà qui peut paraître bien douteux.

Nous avons vu cependant que la Renaissance italienne, et sa puissance créatrice même, a eu pour condition, et peut-être pour signe, l'amour, le désir éperdus de retrouver non pas le passé mort, mais le passé vivant. Cela veut dire qu'une nouvelle Renaissance n'est pas de l'ordre de l'impossible, pourvu qu'on aime avec suffisamment de force les grandes époques de l'bumanité qui nous a précédés. Pourvu qu'on aime sans mesure les cuvres de l'esprit et de l'art humains. Qu'on les aime avec autant de ferveur que la Renaissance a aimé l'Antiquité. Pourvu qu'on sache, qu'on sente que le passé n'est pas la mort, et que notre présent, lui, mourrait d'oublier cette évidence. Pourvu qu'on prenne conscience que le présent n'est rien sinon le temps si court qui nous est imparti pour recueillir la vie du passé et l'offrir au futur. Cela s'appelle créer. À ce prix, oui, nous sommes en droit d'espérer.

Étienne Barilier 
* Ce texte est celui d'une conférence donnée au Musée Barbier-Mueller de Genève le 28 février 2017, sous le titre : La Renaissance italienne ou l'amour créateur. Nous lui avons conservé son caractère oral.

I. Cf. A.V. van Stekelenburg, De puella Romae reperta - A Renaissance poem, dans «Akroterion», XXXIX, I 994, p. I33.

2. Ou Fonzio (I 446-I 5 I 3 ).

3. «Facies [...] erat subpallida, ac si eodem die puella sepulta esset. [...] Ad summam et formosissima simul et generosissima haec puella florente adhuc Roma urbe apparet». Cité dans Chr. Imbert, La Belle qui sort du Tombeau: une figure de la Renaissance en Italie et en France, dans «Réforme, Humanisme, Renaissance», $\mathrm{n}^{\circ} 75$, 20I 2, p. I 45 .

4. «Et erat adea pulchra et formosa, quod vix scribi seu dici posset, et si diceretur aut scriberetur, a legentibus qui eam non viderunt minime crederetur». Texte de Stefano Infessura, cité in $\mathrm{H}$. Thode, Die römische Leiche vom Jahre 1485. Ein Beitrag zur Geschichte der Renaissance, dans «Mitteilungen des Instituts für Österreichische Geschichtsforschung», 4, I883, pp. 82-83, note 2. Cf. aussi H. Janitschek, Die Gesellschaft der Renaissance in Italien, Stuttgart, W. Spermann, I 879, pp. $9^{8}$ et I 20.

5. Cf. van Stekelenburg, De puella Romae reperta - A Renaissance poem, cit., p. I 35.

6. Même si l'on sait qu'effectivement les Romains maîtrisaient ces techniques. Cf. à ce sujet $\mathrm{H}$. Thode, Die römische Leiche vom Jahre 1485 , cit., p. 89, note I.

7. Cf. J. Burckhardt, Civilisation de la Renaissance en Italie, Le Livre de Poche Illustré, vol. II, I966, pp. 3 I-32.

8. Cf. van Stekelenburg, De puella Romae reperta - A Renaissance poem, cit., p. I 37. Il s'agit d'un poème de Conradus Celtis.

9. Cf. van Stekelenburg, De puella Romae reperta - A Renaissance poem, cit., p. I 36. Le dessin dans Codex Ashmolensis, Oxford, Bodleian Library, Ms lat. misc. d 85 fol. I6I $v$. (Roman) est consultable sur le site de la Bodleian Library: http://bodley3o.bodley.ox.ac.uk:8 I 80/luna/servlet/detail/ODLodl I I 46394 I I 7779:Epigraphical-and-archaeological-col?sort=Shelfmark $\% 2 \mathrm{CFolio}$ - Page $\%{ }_{2}$ CRoll- $\%{ }_{2} 3 \%{ }_{2} \mathrm{CFrame}-\%{ }_{2} 3$ \&qvq $=\mathrm{q}:$ ashmolensis;sort:Shelfmark $\%{ }_{2} \mathrm{C}$ Folio-Page $\%{ }_{2}$ CRoll- $\% 223 \% 2$ CFrame- $\%{ }_{2} 3 ;$ lc:ODLodl $\sim$ I $\sim$ I \& mi $=47 \&$ trs $=49 . \quad$ Il est en outre reproduit dans F. Saxl, The Classical Inscription in Renaissance Art and Politics. Bartolommeus Fontius, Liber Monumentorum Romanae Urbis et aliorum locorum, dans «Journal of the Warburg Institute», IV, I-2, oct. I940-janv. 4I, pp. 19-46. Un autre dessin, passablement différent, tiré du Codex Ashburnham, i I 74, f. I 34, se trouve reproduit dans R. Lanciani, Pagan and Christian Rome, Boston et New York, Houghton, Mifflin and Company, I 892, p. 298.

ı०. «Platonis imaginem diu, multumque desideraverat. Hanc tandem in ipsis Academiae ruinis repertam quum a Hieronymo Roscio Pistoriensi accepisset gaudio exultavit, at Marsilium dicentem audiri, eamque summa semper in vene- 
ratione habuit». (cf. Niccolò Valori, Laurentii Medices vita, in Pbilippii Villani liber de civitatis Florentiaie famosis civibus, Florentiae, Joannes Mazzoni excudebat, MDCCCXLVII, p. I68. Anecdote évoquée par A. Chastel, Art et humanisme à Florence au temps de Laurent le Magnifique, Paris, Presses Universitaires de France, I 959, p. 72, note I, avec une leçon fautive).

I I. Voici deux de ces vers, tirés de sa réponse trop modeste à son admirateur. En français, le sens de ce texte est à peu près le suivant: «Mes vers, honnêtement ne sont qu'essais joueurs / Essais de jeune fille, simples fleurs et rosée». En grec :

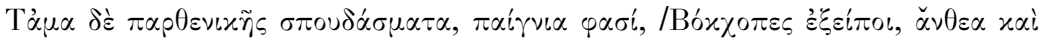

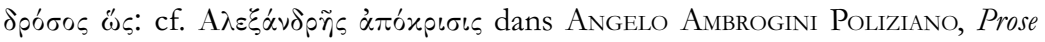
volgari inedite e poesie latine e greche edite e inedite, Firenze, G. Barbèra Editore, i 867, p. 202. Bocchyris était un juge égyptien incorruptible, devenu symbole du jugement équitable.

I 2. Dans ses Commentarii de 1436, éd. moderne, Florence, Giunti, 1986. Cité dans Dictionnaire culturel de la langue française, article «mimésis».

I 3. Cité in Chastel, Art et humanisme à Florence cit., p. $42 \mathrm{I}$.

14. Cf. Burckhardt, Civilisation de la Renaissance en Italie, vol. II, pp. 286-87.

I 5. «Nec certam sedem, nec propriam faciem, nec munus ullum peculiare tibi dedimus, o Adam, ut quam sedem, quam faciem, quae munera tute optaveris, ea, pro voto, pro tua sententia, habeas et possideas. Definita caeteris natura intra praescriptas a nobis leges cohercetur. Tu, nullis angustiis cohercitus, pro tuo arbitrio, in cuius manu te posui, tibi illam prefinies. Medium te mundi posui, ut circumspiceres inde comodius quicquid est in mundo. Nec te celestem neque terrenum, neque mortalem neque immortalem fecimus, ut tui ipsius quasi arbitrarius honorariusque plastes et fictor, in quam malueris tute formam effingas. Poteris in inferiora quae sunt bruta degenerare; poteris in superiora quae sunt divina ex tui animi sententia regenerari».

16. Cité dans H. de Lubac, Pic de la Mirandole, Paris, Aubier-Montaigne, i974, pp. 170-7I.

17. Cf. Sophocle, Antigone, v. 332 ss, trad. de Paul Mazon, coll. Classiques en Poche, les Belles-Lettres, Paris, 2002, pp. 28-29.

I 8. De savants comparatistes ont même pu établir que le balancement du texte de Pic, décrivant l'homme comme alternativement et également capable du bien et du mal, reprend, à la fois pour le rythme et pour le sens, celui du texte de Sophocle. Cf. M. Mastroianni, Le Antigoni sofoclee del Cinquecento francese, Firenze, Olschki, Firenze, 2004, p. 2 I 9.

I9. Ces mots ne sont pas superposables à ceux que l'on trouve sous la plume de Nicolas de Cues dans un texte de I44I: «L'homme peut être ange humain, bête humaine [...] ou quoi que ce soit d'autre». Cf. Nicolas de Cues, De Conjecturis, II, I4, cité par E. Cassirer, Individu et cosmos dans la philosophie de la Renaissance, Paris, Minuit, I983, p. I 5 . 


\section{ÉtIENNE BARILIER}

20. Pascal, dans sa fameuse description de l'homme «ni ange ni bête», en conclura qu'il est alors «un monstre incompréhensible».

2 I. Cf. J. Huizinga, L'automne du Moyen-Âge, ch. intitulé «La vision de la mort». 